\title{
THE INCONSISTENCY PREDICATE ON DE MORGAN LATTICES
}

\author{
ADAM PŘENOSIL \\ Department of Logic, Faculty of Arts, Charles University \\ E-mail: adam.prenosil@gmail.com
}

\begin{abstract}
We consider expansions of De Morgan lattices by an additional unary predicate interpreted in each De Morgan lattice by the ideal generated by all elements of the form $a \wedge-a$, and describe the finite lattice of strict universal Horn classes of such structures, thereby extending the description of the lattice of quasivarieties of De Morgan lattices due to Pynko. We also consider the same problem for expansions of De Morgan lattices by a constant interpreted as the maximal element of this ideal whenever it exists.

Keywords: De Morgan lattice, inconsistency, Belnap-Dunn logic, quasivariety, universal Horn class
\end{abstract}

\section{Introduction}

The present paper is a logically motivated investigation of certain expansions of De Morgan lattices. Before dealing with the technical side of the matter, let us therefore first outline the logical motivation.

The four-valued Belnap-Dunn logic $[2,4]$ was devised to handle inferences from inconsistent premises in a non-trivial way. It validates double negation introduction and elimination and the De Morgan laws without validating either the law of excluded middle or ex contradictione quodlibet. The Belnap-Dunn logic has a transparent four-valued semantics which preserves the truth and falsehood conditions of classical logic but allows propositions to be both true and false (corresponding to inconsistent information) or neither true nor false (corresponding to incomplete information). An essential feature of this logic is that consistent and inconsistent theories are treated on a par.

An algebraic semantics of the four-valued Belnap-Dunn logic is provided by De Morgan lattices, i.e. distributive lattices equipped with an order-inverting involution, called the De Morgan negation here. Each De Morgan lattice may be interpreted as an algebra of propositions where the lattice ordering corresponds to the entailment relation between propositions. Then $\Gamma \vdash \Delta$ holds in the Belnap-Dunn logic if and only if the inequality $\bigwedge \Gamma \leq \bigvee \Delta$ holds in each De Morgan lattice.

If we now broaden our notion of logic slightly to subsume inferences between sequents rather than formulas, we may say that the sequent $\Gamma \vdash \Delta$ is a consequence of the sequents $\Gamma_{1} \vdash \Delta_{1}, \ldots, \Gamma_{n} \vdash \Delta_{n}$ if and only if the quasiequation $\bigwedge \Gamma_{1} \leq \bigvee \Delta_{1} \& \ldots \& \wedge \Gamma_{n} \leq$ 
$\bigvee \Delta_{n} \Rightarrow \bigwedge \Gamma \leq \bigvee \Delta$ holds in each De Morgan lattice. That is, the Belnap-Dunn logic in this broader sense may be identified with the quasiequational theory of De Morgan lattices.

Our intention is now to extend the expressive power of the Belnap-Dunn logic (in this broader sense) by talking not only about which propositions follow from which, but also about which propositions are inconsistent. To this end we allow the premises and conclusions of our inferences to be not only sequents, but also statements of the form "the proposition $\varphi$ inconsistent". This expansion of the Belnap-Dunn logic then allows us to express principles such as "if $\varphi \wedge \psi$ is inconsistent and $-\psi$ is inconsistent, then $\varphi$ is inconsistent" or "if $\varphi$ is inconsistent and $-\psi$ is inconsistent, then $\varphi$ entails $\psi$ ".

It remains to specify what we mean by calling a proposition inconsistent. There are broadly two diferent options to choose from: either we talk about logical inconsistency or about material inconsistency. In the former interpretation, a proposition is inconsistent by virtue of its logical form, e.g. all propositions of the form $(\varphi \wedge-\varphi) \vee(\psi \wedge-\psi)$ are logically inconsistent. In the latter interpretation, it is allowed that there may be propositions which are inconsistent but not merely by virtue of their logical form, i.e. inconsistency is treated as a primitive notion. These interpretations correspond to two notions of an inconsistent predicate on a De Morgan lattice: the standard inconsistency predicate, which is precisely the ideal generated by elements of the form $a \wedge-a$, and an arbitrary inconsistency predicate, which is any ideal extending the standard inconsistency predicate.

The main question which we answer here is: how many strict universal Horn classes of De Morgan lattices expanded by the standard inconsistency predicate (i.e. a unary predicate interpreted on each De Morgan lattice as the ideal generated by elements of the form $a \wedge-a)$ are there? ${ }^{1}$ For De Morgan lattices, this question was already answered by Pynko [7], who proved that there are only finitely many strict universal Horn classes (i.e. quasivarieties) of De Morgan lattices, and provided a full description of the finite lattice of quasivarieties of De Morgan lattices. By contrast, it was proved by Adams and Dziobiak [1] that there is a continuum of quasivarieties of De Morgan algebras, i.e. De Morgan lattices with a bottom and top element which are part of the algebraic signature of such lattices. In this paper, we extend Pynko's result and show that in this respect De Morgan lattices expanded by the standard inconsistency predicate behave like De Morgan lattices rather than De Morgan algebras, i.e. we prove that there are only finitely many strict universal Horn classes of such structures.

The structure of the paper is as follows. After reviewing some known facts about De Morgan lattices in Section 2, we properly define the notions sketched above and introduce several classes of De Morgan lattices with an inconsistency predicate (called De Morgan $\mathcal{J}$-lattices throughout the paper for simplicity) in Section 3. The main result of Section 4 then states that these classes in fact exhaust all of the classes which are definable by means of strict universal Horn sentences with the help of the standard inconsistency predicate. In Section 4 we also consider expansions of De Morgan lattices by an inconsistency constant (called De Morgan 0-lattices for simplicity), which is interpreted as the maximal element of the inconsistency predicate whenever it exists. It is proved that there are only

1 Recall that a strict universal Horn class is a class defined by strict universal Horn sentences, i.e. disjunctions of finitely many negated atomic formulas (possibly none) and a single atomic formula in a given signature, which may in general contain relational symbols other than the equality sign. 
Figure 1. Some De Morgan lattices

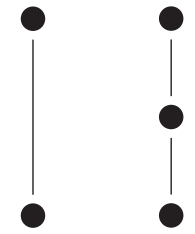

$\mathbf{B}_{2}$

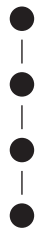

$\operatorname{RegK}_{4}$

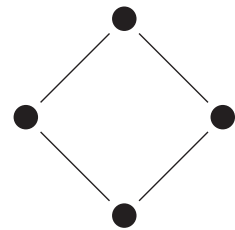

$\mathrm{DM}_{4}$

finitely many strict universal Horn classes of Kleene lattices (but not of De Morgan lattices) with a standard inconsistency constant. The paper then concludes with some open questions.

\section{Preliminaries}

A De Morgan lattice is a distributive lattice equipped with an order-inverting involution, i.e. a unary operation denoted - which satisfies the equations $--x=x$ and $-(x \vee y)=-x \wedge-y$, or equivalently $-(x \wedge y)=-x \vee-y$.

Figure 1 depicts some important De Morgan lattices. In all cases the De Morgan negation consists in turning the lattice upside down around the horizontal axis. In particular, note that $\mathbf{D M}_{\mathbf{4}}$ is not isomorphic as a De Morgan lattice to $\mathbf{B}_{\mathbf{2}} \times \mathbf{B}_{\mathbf{2}}$. The algebras $\mathbf{B}_{\mathbf{2}}, \mathbf{K}_{\mathbf{3}}$, and $\mathbf{D M}_{\mathbf{4}}$ are the only three subdirectly irreducible De Morgan lattices.

Definition 2.1. A De Morgan lattice is Boolean if it satisfies the equation $x \wedge-x \leq y$, and it is Kleene if it satisfies the equation $x \wedge-x \leq y \vee-y$.

Definition 2.2. A De Morgan lattice is non-idempotent if it satisfies the quasiequation $x=-x \Rightarrow x=y$, and it is Kleene-regular if it satisfies the quasiequation $x \leq-x \&-x \wedge y \leq$ $x \vee-y \Rightarrow y \leq-y$.

Here we follow the terminology introduced by Pynko [7], except what he calls regular Kleene lattices we shall (temporarily) call Kleene-regular lattices. We shall show later that being Kleene-regular is equivalent to being Kleene and satisfying a condition which we shall call regularity.

The following two facts will be useful later. The first one is originally due to Belnap and Spencer [3]. It was also proved as Lemma 4.3 in [7]. The second one was established in the course of the proof of Theorem 4.8 of [7].

Lemma 2.3. A non-trivial De Morgan lattice $\mathbf{A}$ is non-idempotent if and only if there is a De Morgan lattice homomorphism $h: \mathbf{A} \rightarrow \mathbf{B}_{2}$.

Lemma 2.4. If a De Morgan lattice $\mathbf{A}$ is neither Kleene nor non-idempotent, then $\mathbf{D M}_{\mathbf{4}}$ is a subalgebra of $\mathbf{A}$. 
Figure 2. The lattice of quasivarieties of De Morgan lattices

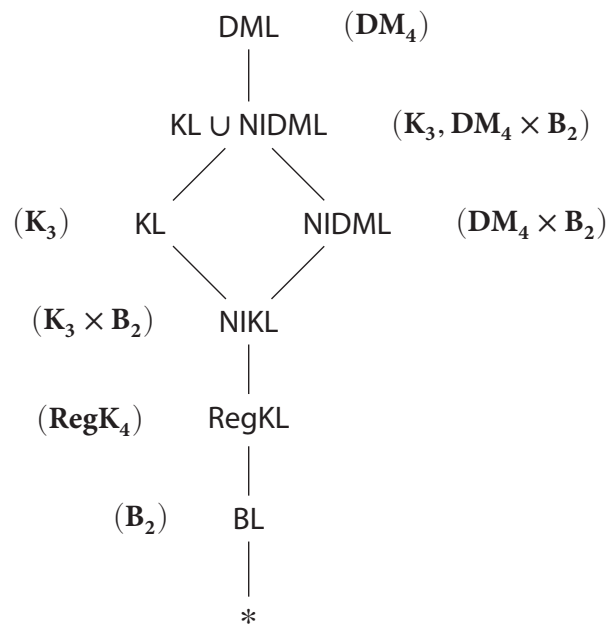

A strict universal Horn sentence is a disjunction of finitely many negated atomic formulas (possibly none) and exactly one atomic formula. A strict universal Horn class is then a class of similar structures axiomatized by a set of strict universal Horn sentences. Equivalently (see [6]), a strict universal Horn class is a similar class of structures closed under substructures and non-empty products and ultraproducts which contains the trivial structure (the singleton structure in which each atomic predicate is interpreted by a non-empty relation). We follow Gorbunov [6] in using the term quasiequation for strict universal Horn sentences and the terms quasiequational class or quasivariety for strict universal Horn classes, even in a signature which contains relational symbols other than the equality sign.

Note that, like Pynko [7], we shall in fact not rely on the assumption of finitarity and closure under ultraproducts. Instead of strict universal Horn sentences, we could therefore discuss their infinitary counterparts, and instead of strict universal Horn classes, we could talk about implicational classes in essentially the same sense as Pynko. The word "quasivariety" may thus be replaced by "implicational class" throughout the paper. For the sake of its familiarity, however, we stick to the language of quasivarieties.

The lattice of quasivarieties of De Morgan lattices was described by Pynko [7], who also showed that each implicational class of De Morgan lattices is a quasivariety. This lattice is shown in Figure 2 along with the generators of each quasivariety. The notation BL, RegKL, NIKL, KL, NIDML, and DML refers to the classes of Boolean lattices, Kleene-regular lattices, non-idempotent Kleene lattices, Kleene lattices, non-idempotent De Morgan lattices, and De Morgan lattices, respectively. The class KL $\cup$ NIDML is a quasivariety axiomatized by the quasiequation $x=-x \Rightarrow y \wedge-y \leq z \vee-z$, and the node labelled $*$ corresponds to the trivial quasivariety. 
Finally, let us emphasize the distinction between De Morgan lattices and De Morgan algebras. A De Morgan algebra is a De Morgan lattice with a bottom and top element which are part of the algebraic signature. Each finite De Morgan lattice may thus be expanded to a De Morgan algebra. However, as noted in the introduction, this seemingly innocent addition of the bottom and top elements to the signature has a dramatic impact on the lattice of quasivarieties: there is a continuum of quasivarieties of De Morgan (in fact, Kleene) algebras but there are only finitely many quasivarieties of De Morgan lattices. We emphasize that throughout the present paper we deal with De Morgan lattices. The only results which in any way involve De Morgan algebras and their expansions are Propositions 4.6 and 4.9.

\section{The inconsistency predicate}

As we already observed in the introduction, each De Morgan lattice A comes equipped with an ideal, denoted $\mathcal{J}_{\mathbf{A}}$, generated by the elements of the form $a \wedge-a$ for $a \in \mathbf{A}$, or equivalently by the elements $a \in \mathbf{A}$ such that $a \leq-a$. If $\mathbf{A}$ is Kleene, then the ideal $\mathcal{J}_{\mathbf{A}}$ admits a particularly simple description.

Lemma 3.1. If $\mathbf{A}$ is a Kleene lattice, then $\mathcal{J}_{\mathbf{A}}=\{a \in \mathbf{A} \mid a \leq-a\}$.

Proof. Clearly $\{a \in \mathbf{A} \mid a \leq-a\} \subseteq \mathcal{J}_{\mathbf{A}}$. Conversely, suppose that $a=\left(a_{1} \wedge-a_{1}\right) \vee$ $\ldots \vee\left(a_{n} \wedge-a_{n}\right) \not \leq\left(a_{1} \vee-a_{1}\right) \wedge \ldots \wedge\left(a_{n} \vee-a_{n}\right)=-a$ for some $a \in \mathbf{A}$. Then there are $a_{i}$ and $a_{j}$ such that $a_{i} \wedge-a_{i} \not \leq a_{j} \vee-a_{j}$, hence $\mathbf{A}$ is not Kleene.

If $\mathbf{A}$ is a De Morgan lattice and $\mathcal{J}$ is an ideal on $\mathbf{A}$ such that $\mathcal{J}_{\mathbf{A}} \subseteq \mathcal{J}$, then the structure $(\mathbf{A}, \mathcal{J})$ will be called a De Morgan lattice with an inconsistency predicate or a De Morgan $\mathcal{J}$ lattice for short. De Morgan $\mathcal{J}$-lattices form a quasivariety which is axiomatized relative to the quasivariety of De Morgan lattices by the quasiequations $a \in \mathcal{J} \& b \in \mathcal{J} \Rightarrow a \vee b \in \mathcal{J}$ and $a \wedge-a \in \mathcal{J}$.

A De Morgan $\mathcal{J}$-lattice $(\mathbf{A}, \mathcal{J})$ will be called standard if $\mathcal{J}=\mathcal{J}_{\mathbf{A}}$, and it will be called totally inconsistent if the ideal $\mathcal{J}$ is the whole of $\mathbf{A}$. Each De Morgan lattice therefore has a unique standard expansion and a unique totally inconsistent expansion.

We shall now define several quasivarieties of De Morgan $\mathcal{J}$-lattices and show that they correspond, in a natural sense, to the quasivarieties of De Morgan lattices defined in the previous section.

Definition 3.2. A De Morgan J-lattice is:

- Boolean if it satisfies $x \in \mathcal{J} \Rightarrow x \leq y$

- Kleene if it satisfies $x \in \mathcal{J} \&-y \in \mathcal{J} \Rightarrow x \leq y$

- non-idempotent if it satisfies $x \in \mathcal{J} \&-x \in \mathcal{J} \Rightarrow x=y$

- regular if it satisfies $x \wedge y \in \mathcal{J} \&-y \in \mathcal{J} \Rightarrow x \in \mathcal{J}$

Proposition 3.3. Each Kleene J-lattice is standard.

Proof. Suppose that $a \in \mathcal{J}$ holds in a Kleene $\mathcal{J}$-lattice $(\mathbf{A}, \mathcal{J})$. Then clearly $-(-a) \in \mathcal{J}$, therefore by the definition of a Kleene $\mathcal{J}$-lattice we have $a \leq-a$, i.e. $a=a \wedge-a \in \mathcal{J}_{\mathbf{A}}$. 
Proposition 3.4. A non-trivial De Morgan J-lattice is totally inconsistent if and only if it is regular but not non-idempotent.

Proof. Each totally inconsistent De Morgan $\mathcal{J}$-lattice $(\mathbf{A}, \mathcal{J})$ is clearly regular, and if it is non-idempotent, then it must be trivial, since $a \in \mathcal{J}$ and $-a \in \mathcal{J}$ for all $a \in \mathbf{A}$. Conversely, if $(\mathbf{A}, \mathcal{J})$ is not non-idempotent, then is some $a \in \mathbf{A}$ such that both $a \in \mathcal{J}$ and $-a \in \mathcal{J}$. If $(\mathbf{A}, \mathcal{J})$ is moreover regular, then taking $y=a$ in the regularity quasiequation yields that the De Morgan $\mathcal{J}$-lattice $(\mathbf{A}, \mathcal{J})$ is totally inconsistent.

The correspondence between De Morgan lattices and their standard expansions is immediate for Boolean, Kleene, and non-idempotent lattices.

Proposition 3.5. A De Morgan lattice is Boolean (Kleene, non-idempotent) if and only if its standard expansion is Boolean (Kleene, non-idempotent).

Proof. If $\mathbf{A}$ is Boolean, then $\mathcal{J}_{\mathbf{A}}=\{\perp\}$, hence $a \in \mathcal{J}_{\mathbf{A}}$ implies $a \leq b$. Conversely, if $\mathcal{J}_{\mathbf{A}}=\{\perp\}$, then $a \wedge-a \leq b$ since $a \wedge-a \in \mathcal{J}_{\mathbf{A}}$ for all $a, b \in \mathbf{A}$.

If $\mathbf{A}$ is Kleene, then by Lemma 3.1 the condition $a \in \mathcal{J}_{\mathbf{A}}$ and $-b \in \mathcal{J}_{\mathbf{A}}$ implies that $a \leq-a$ and $-b \leq b$, hence $a=a \wedge-a \leq b \vee-b=b$. Conversely, if $a \in \mathcal{J}_{\mathbf{A}}$ and $-b \in \mathcal{J}_{\mathbf{A}}$ together imply that $a \leq b$, then in particular $a \wedge-a \leq b \vee-b$ holds for all $a, b \in \mathbf{A}$.

If the standard expansion of $\mathbf{A}$ is non-idempotent and $\mathbf{A}$ is non-trivial, then clearly the De Morgan negation on $\mathbf{A}$ cannot have any fixpoint. Conversely, suppose that $\mathbf{A}$ is a nontrivial non-idempotent De Morgan lattice. Then there is a homomorphism $h: \mathbf{A} \rightarrow \mathbf{B}_{\mathbf{2}}$ by Lemma 2.3. But $\mathcal{J} \subseteq h^{-1}\{\perp\}$ and $-\mathcal{J} \subseteq h^{-1}\{T\}$, hence $\mathcal{J} \cap-\mathcal{J}=\varnothing$.

Finally, the reader will notice that the quasivariety of regular De Morgan $\mathcal{J}$-lattices defined above itself does not correspond to any of the quasivarieties of De Morgan lattices listed in the previous section. Nonetheless, we may still define a corresponding class of regular De Morgan lattices and show that its intersection with the variety of Kleene lattices is precisely the quasivariety of regular Kleene lattices introduced by Pynko [7].

Definition 3.6. A De Morgan lattice $\mathbf{A}$ is regular if for each $a \in \mathbf{A}$ such that $a \notin \mathcal{J}_{\mathbf{A}}$ there is a homomorphism $h: \mathbf{A} \rightarrow \mathbf{B}_{2}$ such that $h(a)=\mathrm{T}$.

It is easy to see that this definition may equivalently be stated as follows: a De Morgan algebra $\mathbf{A}$ is regular if and only if $\mathcal{J}_{\mathbf{A}}=\bigcap_{h \in \operatorname{Hom}\left(\mathbf{A}, \mathbf{B}_{2}\right)} h^{-1}\{\perp\}$, where $\operatorname{Hom}\left(\mathbf{A}, \mathbf{B}_{2}\right)$ denotes the set of all homomorphisms $h: \mathbf{A} \rightarrow \mathbf{B}_{2}$.

It will be useful to provide a description of the smallest congruence $\theta$ on a given De Morgan lattice $\mathbf{A}$ such that $\mathbf{A} / \theta$ is a Boolean lattice. We shall call such congruences Boolean. Each De Morgan lattice has a smallest Boolean congruence, namely the De Morgan lattice congruence generated by identifying all elements of $\mathcal{J}_{\mathbf{A}}$.

Lemma 3.7. The smallest Boolean congruence on a De Morgan lattice $\mathbf{A}$ relates $x$ and $y$ if and only if $-a \wedge(x \vee b)=-a \wedge(y \vee b)$ for some $a, b \in \mathcal{J}_{\mathrm{A}}$.

Proof. Let us denote this relation $\theta$. We first verify that it is a De Morgan lattice congruence. It is clear that $\theta$ is reflexive and symmetric. Moreover, if $-a \wedge(x \vee b)=-a \wedge(y \vee b)$ and $-c \wedge(y \vee d)=-c \wedge(z \vee d)$ for $a, b, c, d \in \mathcal{J}_{\mathbf{A}}$, then $-(a \vee c) \wedge(x \vee b \vee d)=$ $-(a \vee c) \wedge(y \vee b \vee d)=-(a \vee c) \wedge(z \vee b \vee d)$ and clearly $a \vee c \in \mathcal{J}_{\mathbf{A}}$ and $b \vee d \in \mathcal{J}_{\mathbf{A}}$. 
The relation $\theta$ is therefore an equivalence relation. It is easy to see that it also respects meets. To prove that it respects De Morgan negation (and therefore also joins), suppose that $-a \wedge(x \vee b)=-a \wedge(y \vee b)$ for $a, b \in \mathcal{J}_{\mathbf{A}}$. Then $(-x \wedge-b) \vee a=(-y \wedge-b) \vee a$, hence $-(b \wedge-a) \wedge(-x \vee a)=-(b \wedge-a) \wedge(-y \vee a)$ and $-x \theta-y$. The relation $\theta$ is therefore a De Morgan lattice congruence.

If $x, y \in \mathcal{J}_{\mathbf{A}}$, then $x \vee y \in \mathcal{J}_{\mathbf{A}}$ and taking $b=x \vee y$ and arbitrary $a \in \mathcal{J}_{\mathbf{A}}$ yields $x \theta y$, therefore $\theta$ is a Boolean congruence. Conversely, each Boolean congruence identifies $x$ and $-a \wedge(x \vee b)$ as well as $y$ and $-a \wedge(y \vee b)$, therefore each Boolean congruence extends $\theta$.

Proposition 3.8. A De Morgan lattice is regular if and only if its standard expansion is regular.

Proof. Suppose that the implication $x \wedge y \in \mathcal{J}_{\mathbf{A}} \&-y \in \mathcal{J}_{\mathbf{A}} \Rightarrow x \in \mathcal{J}_{\mathbf{A}}$ holds and let $\theta$ be the smallest Boolean congruence on $\mathbf{A}$. If $x / \theta=\perp / \theta$, where $\perp / \theta$ is the bottom element of $\mathbf{A} / \theta$, then by the previous lemma there are $a, b \in \mathcal{J}_{\mathbf{A}}$ such that $-b \wedge(x \vee a) \in \mathcal{J}_{\mathbf{A}}$, hence $-b \wedge x \in \mathcal{J}_{\mathbf{A}}$. But then $x \in \mathcal{J}_{\mathbf{A}}$. This shows that $\mathcal{J}_{\mathbf{A}}=\bigcap_{h \in \operatorname{Hom}\left(\mathbf{A}, \mathbf{B}_{2}\right)} h^{-1}\{\perp\}$.

Conversely, if a De Morgan lattice $\mathbf{A}$ is regular, then $x \in \mathcal{J}_{\mathbf{A}}$ if and only if $h(x)=\perp$ for all homomorphisms $h: \mathbf{A} \rightarrow \mathbf{B}_{2}$. But if $h(x \wedge y)=\perp$ and $h(-y)=\perp$, then $h(y)=\mathrm{T}$ and $h(x)=h(x \wedge y)=\perp$.

Proposition 3.9. A De Morgan lattice is both regular and Kleene if and only if it is Kleene-regular, i.e. if and only if it satisfies the quasiequation $x \leq-x \&-x \wedge y \leq x \vee-y \Rightarrow$ $y \leq-y$.

Proof. Let $\mathbf{A}$ be a De Morgan lattice which satisfies the above quasiequation. To prove that $\mathbf{A}$ is Kleene, substitute $x=u \wedge-u$ and $y=(u \wedge-u) \vee(v \wedge-v)$. Then $x=u \wedge-u \leq$ $u \vee-u=-x$ and $-x \wedge y=(u \vee-u) \wedge((u \wedge-u) \vee(v \wedge-v))=(u \wedge-u) \vee((u \vee-u) \wedge(v \wedge-v))$, while $x \vee-y=(u \wedge-u) \vee((u \vee-u) \wedge(v \vee-v))=(u \vee-u) \wedge((u \wedge-u) \vee(v \vee-v))$. Therefore $-x \wedge y \leq x \vee-y$ and $y \leq-y$, i.e. $(u \wedge-u) \vee(v \wedge-v) \leq(u \vee-u) \wedge(v \vee-v)$. It follows that $u \wedge-u \leq v \vee-v$.

To prove that $\mathbf{A}$ is regular, suppose that $a / \theta=\perp / \theta$ for each Boolean congruence $\theta$ on $\mathbf{A}$, where $\perp / \theta$ is the bottom element of $\mathbf{A} / \theta$. We are to show that $a \in \mathcal{J}_{\mathbf{A}}$. Lemma 3.7 then implies that there are $b, c \in \mathcal{J}_{\mathbf{A}}$ such that $-b \wedge(a \vee c) \in \mathcal{J}_{\mathbf{A}}$, hence $-b \wedge a=d \in \mathcal{J}_{\mathbf{A}}$. But then $b \vee d \in \mathcal{J}_{\mathrm{A}}$ and $-(b \vee d) \wedge a \leq d$, hence $-(b \vee d) \wedge a \leq-a \vee b \vee d$. Taking into account that $a \in \mathcal{J}_{\mathbf{A}}$ is equivalent to $a \leq-a$ for all $a \in \mathbf{A}$ by Lemma 3.1, substituting $x=b \vee d$ and $y=a$ into the quasiequation now yields that $a \in \mathcal{J}_{\mathbf{A}}$.

Conversely, let $\mathbf{A}$ be a regular Kleene lattice with $a \leq-a$ and $b \not \leq-b$, i.e. $a \in \mathcal{J}_{\mathbf{A}}$ and $b \notin \mathcal{J}_{\mathbf{A}}$ by Lemma 3.1. By regularity, there is some $h: \mathbf{A} \rightarrow \mathbf{B}_{\mathbf{2}}$ such that $h(b)=\mathrm{T}$. But $h(a)=\perp$ and $h(-a \wedge b)=-h(a) \wedge h(b)=\top \not \leq \perp=h(a) \vee-h(b)=h(a \vee-b)$, hence $-a \wedge b \not \leq \vee \vee-b$.

The above proposition may be thought of as an explanation of the rather non-transparent quasiequation $x \leq-x \&-x \wedge y \leq x \vee-y \Rightarrow y \leq-y$.

We now compile the correspondences proved above into a single theorem. 
Theorem 3.10. If $(\mathbf{A}, \mathcal{J})$ is Boolean (Kleene, non-idempotent), then so is the De Morgan lattice $\mathbf{A}$. Conversely, if $\mathbf{A}$ is Boolean (Kleene, non-idempotent, regular), then so is its standard expansion $\left(\mathbf{A}, \mathcal{J}_{\mathbf{A}}\right)$.

Proof. We have already proved that a De Morgan lattice is Boolean (Kleene, nonidempotent, regular) if and only if it standard expansion is. The first claim of the theorem now follows by virtue of the fact that $\mathcal{J}_{\mathbf{A}} \subseteq \mathcal{J}$ holds in every De Morgan $\mathcal{J}$-lattice $(\mathbf{A}, \mathcal{J})$ and the predicate $\mathcal{J}$ does not occur in the consequent of any of the quasiequations defining the classes of Boolean, Kleene, and non-idempotent De Morgan $\mathcal{J}$-lattices.

Observe that the first part of the above theorem does not hold when it comes to regularity, a simple counter-example being the totally inconsistent expansion of any nonregular De Morgan algebra.

\section{Quasivarieties of De Morgan $\mathcal{J}$-lattices}

We shall now investigate precisely how much expressive power the standard inconsistency predicate adds to De Morgan lattices. The goal of the present section will be to describe the lattice of quasivarieties of standard De Morgan $\mathcal{J}$-lattices, and in particular to prove that it is finite.

Before we can proceed, the notion of a quasivariety of standard De Morgan $\mathcal{J}$-lattices requires some clarification. The class of all standard De Morgan $\mathcal{J}$-lattices is not closed under substructures (consider the two-element subalgebra of the standard expansion of $\mathbf{D M}_{4}$ ), in particular it is not a quasivariety. By a quasivariety of standard De Morgan $\mathcal{J}$ lattices we shall therefore not mean a quasivariety all of whose elements are standard De Morgan $\mathcal{J}$-lattices. Rather, we shall use the following definition.

Definition 4.1. A class of standard De Morgan J-lattices is a quasivariety of standard De Morgan $\mathcal{J}$-lattices if it is the intersection of a quasivariety of De Morgan $\mathcal{J}$-lattices and the class of all standard De Morgan J-lattices.

Quasivarieties of standard De Morgan $\mathcal{J}$-lattices may be put into one-to-one correspondence with certain quasivarieties of De Morgan $\mathcal{J}$-lattices, which are more convenient to handle using the theory of quasivarieties.

Definition 4.2. A quasivariety of De Morgan $\mathcal{J}$-lattices is standard if it is generated by its standard elements.

The quasivarieties of standard De Morgan $\mathcal{J}$-lattices ordered by inclusion form a lattice, as do the standard quasivarieties of De Morgan $\mathcal{J}$-lattices. In the following proposition, $\mathrm{DMIL}_{s t}$ shall denote the class of all standard De Morgan $\mathcal{J}$-lattices.

Proposition 4.3. The lattice of standard quasivarieties of De Morgan J-lattices is isomorphic to the lattice of quasivarieties of standard De Morgan J-lattices via the mapping $\mathrm{K} \mapsto \mathrm{K} \cap \mathrm{DMIL}_{s t}$.

Proof. This mapping is clearly monotonic. Moreover, if $\mathrm{K}$ is a quasivariety of De Morgan $\mathcal{J}$-lattices, then $\mathrm{K} \cap \mathrm{DMIL}_{s t}$ is by definition a quasivariety of standard De Morgan 
TABLE 1. The standard quasivarieties of De Morgan $\mathcal{J}$-lattices

\begin{tabular}{|l|l|l|}
\hline Name & Abbreviation & Generated by \\
\hline Boolean & $\mathrm{BL}$ & $\mathbf{B}_{2}^{\mathbf{s}}$ \\
Kleene & $\mathrm{KL}$ & $\mathbf{K}_{\mathbf{3}}^{\mathbf{s}}$ \\
De Morgan & $\mathrm{DML}$ & $\mathbf{K}_{\mathbf{3}}^{\mathbf{s}}, \mathbf{D M}_{\mathbf{4}}^{\mathbf{s}}$ \\
regular & RegDML & $\mathbf{R e g K}_{4}^{\mathbf{s}}, \mathbf{D} \mathbf{M}_{4}^{\mathbf{s}}$ \\
regular Kleene & RegKL & $\mathbf{R e g K}_{\mathbf{4}}^{\mathbf{s}}$ \\
non-idempotent & $\mathrm{NIDML}$ & $\mathbf{K}_{\mathbf{3}}^{\mathbf{s}} \times \mathbf{B}_{2}^{\mathbf{s}}, \mathbf{D M}_{\mathbf{4}}^{\mathbf{s}} \times \mathbf{B}_{2}^{\mathbf{s}}$ \\
non-idempotent Kleene & $\mathrm{NIKL}$ & $\mathbf{K}_{\mathbf{3}}^{\mathbf{s}} \times \mathbf{B}_{2}^{\mathbf{s}}$ \\
regular non-idempotent & $\operatorname{RegNIDML}$ & $\mathbf{D M}_{4}^{\mathbf{s}} \times \mathbf{B}_{\mathbf{2}}^{\mathbf{s}}$ \\
totally inconsistent & $\mathrm{TIL}$ & $\mathbf{D M}_{\mathbf{4}}^{\mathbf{s}}$ \\
\hline
\end{tabular}

$\mathcal{J}$-lattices. It remains to prove that if $K$ and $L$ are standard quasivarieties of De Morgan $\mathcal{J}$-lattices and $\mathrm{K} \cap \mathrm{DMIL}_{s t} \subseteq \mathrm{L} \cap \mathrm{DMIL}_{s t}$, then $\mathrm{K} \subseteq \mathrm{L}$. Since $\mathrm{K}$ and $\mathrm{L}$ are standard, $\mathrm{K} \cap \mathrm{DMIL}_{s t}$ $\left(\mathrm{L} \cap \mathrm{DMIL}_{s t}\right)$ generates $\mathrm{K}(\mathrm{L})$ as a quasivariety, therefore $\mathrm{K} \cap \mathrm{DMIL}_{s t} \subseteq \mathrm{L} \cap \mathrm{DMIL}_{s t}$ implies $\mathrm{K} \subseteq \mathrm{L}$.

Instead of studying the lattice of quasivarieties of De Morgan lattices with a standard inconsistency predicate, we can therefore investigate the isomorphic lattice of standard quasivarieties of De Morgan lattices. In other words, although we are ultimately interested in studying the standard inconsistency predicate on each De Morgan lattice, admitting non-standard inconsistency predicates will be a useful tool in the study of De Morgan lattices equipped with the standard inconsistency predicate.

As an example illustrating the notion of a standard quasivariety, consider the quasivariety of De Morgan $\mathcal{J}$-lattices axiomatized by $x \wedge-x \leq y$. This class is not a standard quasivariety of De Morgan $\mathcal{J}$-lattices because each standard De Morgan $\mathcal{J}$-lattice which satisfies this equation also satisfies the quasiequation $x \in \mathcal{J} \Rightarrow x \leq y$, which however is not a consequence of $x \wedge-x \leq y$, as witnessed by the totally inconsistent expansion of the Boolean lattice $\mathbf{B}_{2}$. On the other hand, the quasivariety axiomatized by $x \in \mathcal{J} \Rightarrow x \leq y$ is standard, as it is generated by the standard expansion of $\mathbf{B}_{\mathbf{2}}$.

When talking about De Morgan $\mathcal{J}$-lattices, the notation $\mathbf{A}^{\mathbf{s}}$ will be used to denote the expansion of the De Morgan lattice $\mathbf{A}$ by the standard inconsistency predicate, as in Table 1. To avoid unnecessary proliferation of indices, we introduce the harmless convention of using e.g. NIDML to denote either the quasivariety of non-idempotent De Morgan lattices or the quasivariety of non-idempotent De Morgan $\mathcal{J}$-lattices or, later on, the quasivariety of non-idempotent De Morgan 0-lattices. It will always be clear from the context which of these is the intended interpretation of e.g. NIDML.

Note that in this section we shall occasionally use the term "algebra" to refer to De Morgan $\mathcal{J}$-lattices, even though strictly speaking they are not algebras in the sense of universal algebra.

Theorem 4.4. The quasivarieties of De Morgan J-lattices introduced in the previous section are generated by the standard algebras shown in Table 1. 
Proof. It is easy to verify that the algebras listed in Table 1 belong to the appropriate quasivarieties. Conversely, we prove that each finite algebra which belongs to a quasivariety listed in Table 1 embeds into a product of the appropriate algebras. In particular, observe that if a finite De Morgan $\mathcal{J}$-lattice $\mathbf{A}$ is a subdirect product of the algebras $\mathbf{B}_{i}$ for $i \in I$, then the ideal $\mathcal{J}_{\mathbf{A}}$ is a product of the ideals $\mathcal{J}_{\mathbf{B}_{i}}$ for $i \in I$.

Let $\mathbf{A}$ be a non-trivial finite De Morgan $\mathcal{J}$-lattice. As a De Morgan lattice, $\mathbf{A}$ is a subdirect product of $\mathbf{B}_{2}, \mathbf{K}_{3}$, and $\mathbf{D M}_{4}$. Moreover, the non-standard (totally inconsistent) expansions of $\mathbf{B}_{\mathbf{2}}$ and $\mathbf{K}_{\mathbf{3}}$ are subalgebras of the standard expansion of $\mathbf{D M}_{\mathbf{4}}$, therefore $\mathbf{A}$ embeds into a product of the standard expansions of $\mathbf{B}_{2}, \mathbf{K}_{3}$, and $\mathbf{D M}_{4}$.

If $\mathbf{A}$ is Kleene, then $\mathbf{A}$ is standard, hence each factor in the subdirect decomposition of $\mathbf{A}$ is standard. It now suffices to recall that each Kleene (Boolean, regular Kleene, nonidempotent Kleene) lattice embeds into a product of the appropriate De Morgan lattices.

If $\mathbf{A}$ is totally inconsistent, the claim follows immediately from the fact that each De Morgan lattice embeds into some power of $\mathbf{D M}_{4}$.

If $\mathbf{A}$ is non-idempotent, then the subdirect decomposition of $\mathbf{A}$ contains $\mathbf{B}_{2}$. If all occurrences of $\mathbf{B}_{2}$ in the subdirect decomposition of $\mathbf{A}$ were totally inconsistent, then A could not be non-idempotent as a De Morgan $\mathcal{J}$-lattice. Therefore $\mathbf{A}$ contains an occurrence of $\mathbf{B}_{2}^{s}$ in its subdirect decomposition. The claim now follows by virtue of the inclusions $\mathbf{K}_{3}^{\mathrm{ti}} \times \mathbf{B}_{2}^{\mathrm{s}} \subseteq \mathbf{D M}_{4}^{\mathrm{s}} \times \mathbf{B}_{2}^{\mathrm{s}}$ and $\mathbf{B}_{2}^{\mathrm{ti}} \times \mathbf{B}_{2}^{\mathrm{s}} \subseteq \mathbf{D M}_{4}^{\mathrm{s}} \times \mathbf{B}_{2}^{\mathrm{s}}$ and $\mathbf{B}_{2}^{\mathrm{s}} \subseteq \mathbf{K}_{3}^{\mathrm{s}} \times \mathbf{B}_{2}^{\mathrm{s}}$, where $\mathbf{B}_{2}^{\mathrm{ti}}$ and $\mathbf{K}_{3}^{\text {ti }}$ are the totally inconsistent expansions of the De Morgan lattices $\mathbf{B}_{\mathbf{2}}$ and $\mathbf{K}_{\mathbf{3}}$.

Finally, let $\mathbf{A}$ be regular. If $\mathbf{B}_{2}^{\mathbf{s}}$ does not occur in the subdirect decomposition of $\mathbf{A}$, then A is not non-idempotent, hence it is totally inconsistent and embeds into some power of $\mathbf{D M}_{\mathbf{4}}^{\mathbf{s}}$. We may divide the subdirect factors of $\mathbf{A}$ into two groups (each of them possibly empty) and view $\mathbf{A}$ as a subdirect product of a totally inconsistent algebra $\mathbf{B}$ and a Kleene algebra $\mathbf{C}$. Observe now that $\mathbf{A}$ is regular only if $\mathbf{C}$ is: if $a$ witness to the failure of regularity in $\mathbf{C}$, then $(a, b)$ is a witness to the failure of regularity in $\mathbf{A}$ for any $(a, b) \in \mathbf{A}$. But we have already seen that the quasivariety of regular Kleene $\mathcal{J}$-lattices is generated by $\operatorname{RegK}_{4}^{\mathrm{s}}$.

Moreover, if $\mathbf{A}$ is non-idempotent, then as we observed above, it may contain $\mathbf{B}_{2}^{\mathbf{s}}$ in its subdirect decomposition. Since $\mathbf{B}_{2}^{\mathbf{s}} \subseteq \operatorname{RegK}_{4}^{\mathrm{s}}$, we may take the generators to be $\mathbf{R e g K}_{4}^{\mathrm{s}}$ and $\mathbf{D M}_{4}^{\mathrm{s}} \times \mathbf{B}_{2}^{\mathrm{s}}$.

In particular, all of these quasivarieties are standard. Figure 3 depicts the lattice of these quasivarieties ordered by inclusion. The nodes which are, for the sake of easier readability, labelled $\cup$ or $\cap$ are just the unions of the quasivarieties below and the intersections of the quasivarieties above, and the node labelled $*$ is the trivial quasivariety (containing only the singleton De Morgan $\mathcal{J}$-lattice). In particular, $\mathrm{KL} \cup \mathrm{NIDML}$ is the quasivariety axiomatized by $x \in \mathcal{J} \&-x \in \mathcal{J} \& y \in \mathcal{J} \&-z \in \mathcal{J} \Rightarrow y \leq z$.

We now show that Figure 3 in fact shows all standard quasivarieties of De Morgan $\mathcal{J}$-lattices.

Theorem 4.5. The lattice of standard quasivarieties of De Morgan J-lattices is the finite lattice shown in Figure 3.

Proof. The generating algebras listed in Table 1 witness that all of these quasivarieties are distinct and standard. It suffices to prove the following facts for each non-trivial standard quasivariety K of De Morgan $\mathcal{J}$-lattices: 
FIgURE 3. The standard quasivarieties of De Morgan $\mathcal{J}$-lattices

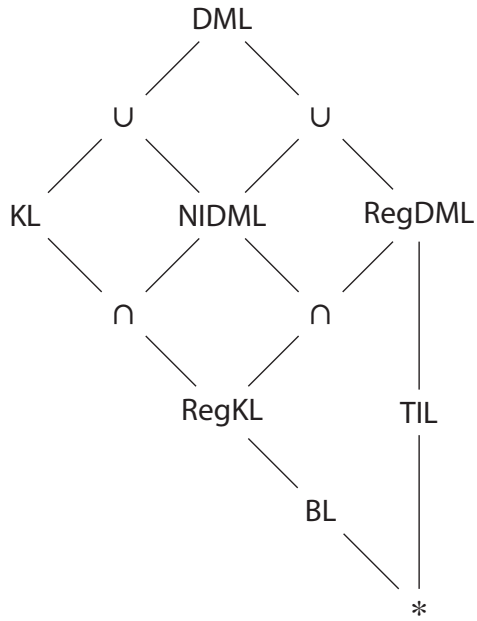

(i) either $\mathrm{BL} \subseteq \mathrm{K}$ or $\mathrm{TIL} \subseteq \mathrm{K}$

(ii) either $\mathrm{BL} \subseteq \mathrm{K}$ or $\mathrm{K} \subseteq \mathrm{TIL}$

(iii) either $\mathrm{K} \subseteq \mathrm{BL}$ or $\mathrm{K} \subseteq \mathrm{TIL}$ or $\operatorname{RegKL} \subseteq \mathrm{K}$

(iv) either $\mathrm{K} \subseteq \mathrm{NIDML} \cup \operatorname{RegDML}$ or $\mathrm{KL} \subseteq \mathrm{K}$

(v) either $\mathrm{K} \subseteq$ RegDML or NIKL $\subseteq \mathrm{K}$

(vi) either $\mathrm{K} \subseteq \mathrm{KL}$ or $\mathrm{K} \subseteq$ TIL or RegNIDML $\subseteq \mathrm{K}$

(vii) either $\mathrm{K} \subseteq$ NIDML $\cup \mathrm{KL}$ or $\mathrm{TIL} \subseteq \mathrm{K}$

To prove (i), let $\mathbf{A}$ be a non-trivial standard De Morgan $\mathcal{J}$-lattice. If $\mathbf{A}$ is not totally inconsistent, then $\mathbf{B}_{2}^{\mathbf{s}} \subseteq \mathbf{A}$. If $\mathbf{A}$ is totally inconsistent, then it is clearly neither Kleene nor non-idempotent, hence $\mathbf{D M}_{\mathbf{4}}^{\mathrm{s}} \subseteq \mathbf{A}$ by Lemma 2.4. Therefore either $\mathrm{BL} \subseteq \mathrm{K}$ or TIL $\subseteq \mathrm{K}$.

To prove (ii), suppose that $\mathrm{K} \nsubseteq \mathrm{TIL}$. Then some $\mathbf{A} \in \mathrm{K}$ is not totally inconsistent. But then $a \notin \mathcal{J}_{\mathbf{A}}$ for some $a \in \mathbf{A}$, hence $\{a \wedge-a, a \vee-a\}$ is a subalgebra of $\mathbf{A}$ isomorphic to

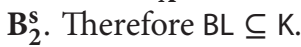

To prove (iii), suppose that $\mathrm{K} \nsubseteq \mathrm{TIL}$ and $\mathrm{K} \nsubseteq \mathrm{BL}$. Then some $\mathbf{A} \in \mathrm{K}$ is not totally inconsistent and some $\mathbf{B} \in \mathrm{K}$ is not Boolean. Let $a \in \mathbf{A}$ be such that $-a \leq a$ and $a \notin \mathcal{J}_{\mathbf{A}}$ (hence $-a<a$ ) and let $b, c \in \mathbf{B}$ be such that $b \leq-b, b \in \mathcal{J}_{\mathbf{B}}$, and $c<b$. Then $\{(-a, c),(-a, b),(a,-b),(a,-c)\}$ is a subalgebra of $\mathbf{A} \times \mathbf{B}$ isomorphic to $\mathbf{R e g K}_{4}^{\mathbf{s}}$. Therefore RegKL $\subseteq \mathrm{K}$.

To prove (iv), suppose that $\mathrm{K} \nsubseteq$ NIDMLURegDML. Then some standard $\mathrm{A} \in \mathrm{K}$ is neither non-idempotent nor totally inconsistent. There is therefore some $a \in \mathbf{A}$ such that $a=-a$ and some $b \in \mathbf{A}$ such that $b \notin \mathcal{J}_{\mathbf{A}}$. Then $\{a \wedge-b, a, a \vee b\}$ is a subalgebra of $\mathbf{A}$ isomorphic to $\mathbf{K}_{\mathbf{3}}^{\mathrm{s}}$. Therefore $\mathrm{KL} \subseteq \mathrm{K}$.

To prove (v), suppose that $\mathrm{K} \nsubseteq \operatorname{RegDML}$. Then $\mathbf{B}_{2}^{\mathrm{s}} \in \mathrm{K}$ and some standard $\mathbf{A} \in \mathrm{K}$ is not regular, i.e. there are some $a \in \mathbf{A}$ and $b \in \mathcal{J}_{\mathbf{A}}$ such that $-b \wedge a \in \mathcal{J}_{\mathbf{A}}$ but $a \notin \mathcal{J}_{\mathbf{A}}$, in particular $a \not \leq-b$. Without loss of generality $b=-b \wedge a$, since $-b \wedge a \in \mathcal{J}_{\mathbf{A}}$ and 
$-(-b \wedge a) \wedge a=(b \vee-a) \wedge a=b \vee(a \wedge-a) \in \mathcal{J}_{\mathrm{A}}$. We may moreover take $a=a \vee b$, since $-b \wedge(a \vee b)=(-b \wedge a) \vee(b \wedge-b) \in \mathcal{J}_{\mathbf{A}}$ and $-b \wedge(a \vee b)=(-b \wedge a) \vee(b \wedge$ $-b)=b \vee(b \wedge-b)=b$. The De Morgan lattice $\{a \wedge-a, b, a,-a,-b, a \vee-a\}$ is a subalgebra of the De Morgan lattice reduct of $\mathbf{A}$, let us call it $\mathbf{B}$. We know that $a \notin \mathcal{J}_{\mathbf{A}}$ and clearly $a \wedge-a, b \in \mathcal{J}_{\mathbf{A}}$. If $-a \not \leq a$, then $\mathbf{B}$ is isomorphic as a De Morgan lattice to $\mathbf{K}_{\mathbf{3}} \times \mathbf{B}_{\mathbf{2}}$, hence the algebra $\mathbf{B} \times \mathbf{B}_{\mathbf{2}}^{\mathbf{s}}$ has a subalgebra isomorphic to $\mathbf{K}_{\mathbf{3}}^{\mathbf{s}} \times \mathbf{B}_{\mathbf{2}}^{\mathbf{s}}$, namely $\{a \wedge-a, b, a\} \times\{\perp\} \cup\{-a,-b, a \vee-a\} \times\{\top\}$. Likewise, if $-a \leq a$, then $-a \leq-b \wedge a=b$, thus $-b \leq a$ and $b=-b \wedge a=-b$. The algebra $\mathbf{B}$ is therefore isomorphic to $\mathbf{K}_{\mathbf{3}}^{\mathbf{s}}$ and $\mathbf{K}_{3}^{\mathbf{s}} \times \mathbf{B}_{\mathbf{2}}^{\mathbf{s}} \in \mathrm{K}$, hence $\mathrm{K} \subseteq \mathrm{NIKL}$.

To prove (vi), suppose that $\mathrm{K} \nsubseteq \mathrm{KL}$ and $\mathrm{K} \nsubseteq \mathrm{TIL}$. Then $\mathbf{B}_{2}^{\mathrm{s}} \in \mathrm{K}$ there is some standard $\mathbf{A} \in \mathrm{K}$ which is not Kleene. Since $\mathbf{A}$ is standard, it is not even Kleene as a De Morgan lattice, i.e. there are $a, b \in \mathbf{A}$ such that $a \leq-a, b \leq-b$, and $a \not \leq-b$. Let $c=a \vee(-a \wedge b)$ and $d=b \vee(-b \wedge a)$. Then $c=a \vee(-a \wedge b) \leq-a \wedge(a \vee-b)=-c$ and likewise $d \leq-d$. Moreover, $c \wedge-d=(a \vee(-a \wedge b)) \wedge-b \wedge(b \vee-a) \leq b \vee(-b \wedge a)=d$ and likewise $-c \wedge d \leq c,-c \leq c \vee-d$, and $-d \leq d \vee-c$. The De Morgan lattice $\{c \wedge d, c, d, c \vee d\}$ is therefore a subalgebra of the De Morgan lattice reduct of $\mathbf{A}$, let us call it $\mathbf{B}$. It is clear that $\{c \wedge d, c, d, c \vee d\} \subseteq \mathcal{J}_{\mathbf{A}}$, hence the algebra $\mathbf{B} \times \mathbf{B}_{2}^{\mathbf{s}}$ has a subalgebra isomorphic to $\mathbf{D M}_{4}^{\text {s }} \times \mathbf{B}_{2}^{\text {s }}$, namely $\{c \wedge d, c, d, c \vee d\} \times\{\perp\} \cup\{-c \wedge-d,-c,-d,-c \vee-d\} \times\{\mathrm{T}\}$. It follows that $\mathrm{K} \subseteq$ RegNIDML.

To prove (vii), suppose that $\mathrm{K} \nsubseteq$ NIDMLUKL. Then there is some standard $\mathbf{A} \in \mathrm{K}$ which is neither Kleene nor non-idempotent. It is therefore neither Kleene nor non-idempotent as a De Morgan lattice, hence $\mathbf{D M}_{\mathbf{4}}^{\mathbf{s}} \subseteq \mathbf{A}$ by Lemma 2.4 and TIL $\subseteq \mathrm{K}$.

In the following proposition, by a De Morgan $\mathcal{J}$-algebra we shall mean a De Morgan $\mathcal{J}$-lattice with a bottom and top element which are part of the signature. The quasivariety of Kleene $\mathcal{J}$-algebras, i.e. De Morgan $\mathcal{J}$-algebras whose appropriate reduct is a Kleene $\mathcal{J}$ lattice, is then denoted $K A$.

Proposition 4.6. Let $\mathrm{K}$ be a quasivariety of De Morgan $\mathcal{J}$-lattices (Jalgebras). Then each algebra in $\mathrm{K}$ is standard if and only if $\mathrm{K} \subseteq \mathrm{KL}(\mathrm{K} \subseteq \mathrm{KA})$.

Proof. Each Kleene $\mathcal{J}$-lattice is standard. Conversely, suppose that $\mathrm{K} \nsubseteq \mathrm{KL}$. Then there is some $\mathbf{A} \in \mathrm{K}$ with $a, b \in \mathcal{J}_{\mathbf{A}}$ such that $a \not \leq-b$ in $\mathbf{A}$, hence $c \not \leq-c$ for $c=a \vee b \in \mathcal{J}_{\mathbf{A}}$. But then either $-c<c$, in which case the non-standard expansion $\mathbf{B}_{\mathbf{2}}$ is a subalgebra of $\mathbf{A}$, or $-c \not \leq c$, in which case a non-standard expansion of $\mathbf{B}_{\mathbf{2}} \times \mathbf{B}_{\mathbf{2}}$ is a subalgebra of $\mathbf{A}$.

The proof for De Morgan $\mathcal{J}$-algebras is analogical, except instead of the non-standard expansions of $\mathbf{B}_{\mathbf{2}}$ and $\mathbf{B}_{\mathbf{2}} \times \mathbf{B}_{\mathbf{2}}$ we may have to take the non-standard expansions of the extensions of these De Morgan lattices by an extra bottom and top element.

Finally, we consider how the expressive power of our language changes when we replace the inconsistency predicate by an inconsistency constant. By a De Morgan lattice with an inconsistency constant (or briefly De Morgan 0-lattice) we shall mean a De Morgan lattice $\mathbf{A}$ equipped with a constant 0 which satisfies the equation $x \wedge-x \leq 0$. $^{2}$ Such an algebra is standard if $\mathcal{J}_{\mathbf{A}}$ is precisely the principal ideal generated by the element 0 . Each finite De Morgan lattice has a unique expansion by a standard inconsistency constant,

2 Do not confuse the constant 0 with the bottom element of the lattice. 
TABLE 2. The quasivarieties of Kleene 0-lattices

\begin{tabular}{|l|l|l|}
\hline Name & Abbreviation & Generated by \\
\hline Boolean & $\mathrm{BL}$ & $\mathbf{B}_{\mathbf{2}}^{\mathbf{s}}$ \\
Kleene & $\mathrm{KL}$ & $\mathbf{B}_{\mathbf{2}}^{\mathbf{s}}, \mathbf{K}_{\mathbf{3}}^{\mathbf{s}}$ \\
regular Kleene & RegKL & $\mathbf{R e g K}_{\mathbf{4}}^{\mathbf{s}}$ \\
non-idempotent Kleene & $\mathrm{NIKL}$ & $\mathbf{K}_{\mathbf{3}}^{\mathbf{s}} \times \mathbf{B}_{\mathbf{2}}^{\mathbf{s}}$ \\
idempotent Kleene & IdemKL & $\mathbf{K}_{\mathbf{3}}^{\mathbf{s}}$ \\
\hline
\end{tabular}

namely $0=\bigvee \mathcal{J}_{\mathbf{A}}$. A quasivariety of De Morgan 0-lattices is again called standard if it is generated by its standard elements.

Each inconsistency constant 0 on $\mathbf{A}$ defines the inconsistency predicate $\{a \in \mathbf{A} \mid a \leq 0\}$ on A. All notions defined in the previous section of De Morgan $\mathcal{J}$-lattices thereby extend to De Morgan 0-lattices. In addition, we shall call a De Morgan 0-lattice idempotent if it satisfies the equation $-0 \leq 0$. We now show that idempotence is the only new property of Kleene lattices which may be expressed quasiequationally using the standard inconsistency constant (rather than the standard inconsistency predicate). By contrast, we show that there are infinitely many standard quasivarieties of De Morgan 0-lattices (although we do not establish exactly how many).

When talking about De Morgan 0-lattices, the notation $\mathbf{A}^{\mathbf{s}}$ will be used to denote the expansion of the De Morgan lattice $\mathbf{A}$ by the standard inconsistency constant (provided that it exists), as in Table 2. Here by a standard inconsistency constant we mean one which generates a standard inconsistency predicate as its principal ideal. Note that the inconsistency constant 0 is standard if and only if there is a finite set $B \subseteq \mathbf{A}$ such that $0=\bigvee_{a \in B}(a \wedge-a)$. It will also be useful to define 1 as -0 .

Theorem 4.7. The quasivarieties of De Morgan 0-lattices introduced in the previous section are generated by the standard algebras shown in Table 2.

Proof. Let $\mathbf{A}$ be a Kleene 0-lattice. Then the Kleene lattice reduct of $\mathbf{A}$ is a subdirect product of copies of $\mathbf{B}_{\mathbf{2}}$ and $\mathbf{K}_{\mathbf{3}}$. Since $0 \leq 1$, the component of 0 in each subdirect factor has to be the standard one on pain of violating the equality $0 \leq 1$, hence $\mathbf{A}$ is a subdirect product of $\mathbf{B}_{2}^{\mathbf{s}}$ and $\mathbf{K}_{\mathbf{3}}^{\mathbf{s}}$. If $\mathbf{A}$ is Boolean, then it is Boolean as a Kleene lattice, hence it is a subdirect power of $\mathbf{B}_{2}^{\mathbf{s}}$. If $\mathbf{A}$ is non-idempotent, i.e. if $1 \not \leq 0$, then at least one of the subdirect factors has to be isomorphic to $\mathbf{B}_{2}^{\mathbf{s}}$ (moreover, $\mathbf{B}_{2}^{\mathbf{s}} \subseteq \mathbf{K}_{3}^{\mathbf{s}} \times \mathbf{B}_{2}^{\mathbf{s}}$ ). If on the other hand $\mathbf{A}$ is idempotent, i.e. if $1=0$, then all of the subdirect factors must be isomorphic to $\mathbf{K}_{\mathbf{3}}^{\mathbf{s}}$. Finally, if $\mathbf{A}$ is regular, i.e. if it satisfies the quasiequation $1 \wedge x \leq 0 \Rightarrow x \leq 0$, then $\mathbf{A}$ is regular as a Kleene lattice, therefore as a Kleene lattice it is a subdirect power of $\mathbf{R e g K}_{\mathbf{4}}$. But then the component of 0 in each subdirect factor again has to be the standard one, therefore $\mathbf{A}$ is a subdirect power of $\operatorname{RegK}_{4}^{\mathrm{s}}$.

We shall now describe the lattice of quasivarieties of Kleene 0-lattices. Note that all such quasivarieties are standard, since each Kleene 0-lattice is easily seen to be standard.

Theorem 4.8. The lattice of quasivarieties of Kleene 0-lattices is the finite lattice shown in Figure 4. 
Figure 4. The quasivarieties of Kleene 0-lattices

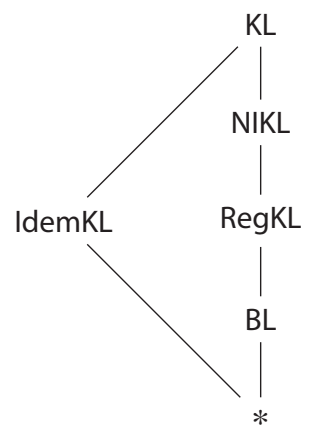

Proof. If $\mathbf{A}$ is a non-trivial Kleene 0-lattice, then either $0<1$, in which case $\mathbf{B}_{2}^{\mathbf{s}} \subseteq \mathbf{A}$, or $0=1$, in which case $\mathbf{K}_{\mathbf{3}}^{\mathbf{s}} \subseteq \mathbf{A}$. Therefore for each non-trivial quasivariety $\mathrm{K}$ of Kleene 0 lattices we have either IdemKL $\subseteq$ K or $\mathrm{BL} \subseteq \mathrm{K}$, and either $\mathrm{K} \subseteq$ IdemKL or $\mathrm{BL} \subseteq \mathrm{K}$. Suppose therefore that $\mathrm{BL} \subseteq \mathrm{K}$, i.e. $\mathbf{B}_{2}^{\mathrm{s}} \in \mathrm{K}$. If $\mathrm{K} \nsubseteq \mathrm{BL}$, then there is some non-Boolean $\mathbf{A} \in \mathrm{K}$, hence $\mathbf{A} \times \mathbf{B}_{2}^{\mathbf{s}}$ is a non-idempotent non-Boolean Kleene 0-lattice and $\operatorname{RegK}_{4}^{s} \subseteq \mathbf{A}$. If K is a quasivariety such that $\mathrm{K} \nsubseteq \operatorname{RegKL}$, then $\mathbf{B}_{2}^{\mathbf{s}} \in \mathrm{K}$ and there is some non-regular $\mathbf{A} \in \mathrm{K}$, i.e there is some $a \in \mathbf{A}$ such that $1 \wedge a \leq 0$ but $a \not \leq 0$. Without loss of generality $a=a \vee 0$, i.e. $0 \leq a$. Then it is straightforward to verify that $\{a \wedge-a, 0, a,-a, 1, a \vee-a\}$ is a subalgebra of $\mathbf{A}$ isomorphic to $\mathbf{K}_{\mathbf{3}}^{\mathbf{s}} \times \mathbf{B}_{\mathbf{2}}^{\mathbf{s}}$. Finally, if $\mathrm{K} \nsubseteq \mathrm{NIKL}$, then there is some non-trivial $\mathbf{A} \in \mathrm{K}$ which is not non-idempotent, hence $\mathbf{K}_{\mathbf{3}}^{\mathbf{s}} \subseteq \mathbf{A}$.

In the following proposition, by a De Morgan 0-algebra we shall mean a De Morgan 0 -lattice with a bottom and top element which are part of the signature. The quasivariety of Kleene 0-algebras, i.e. De Morgan 0-algebras whose appropriate reduct is a Kleene 0lattice, is then denoted $\mathrm{KA}$.

Proposition 4.9. Let $\mathrm{K}$ be a quasivariety of De Morgan 0-lattices (0algebras). Then each algebra in $\mathrm{K}$ is standard if and only if $\mathrm{K} \subseteq \mathrm{KL}(\mathrm{K} \subseteq \mathrm{KA})$.

Proof. Each Kleene 0-lattice is standard. Conversely, let $\mathrm{K}$ be a quasivariety of De Morgan 0 -lattices such that $\mathrm{K} \nsubseteq \mathrm{KL}$. Then there is some $\mathbf{A} \in \mathrm{K}$ such that $0 \not \leq 1$ in $\mathbf{A}$. But then either $1<0$, in which case the non-standard expansion of $\mathbf{B}_{2}$ is a subalgebra of $\mathbf{A}$, or $1 \not \leq 0$, in which case a non-standard expansion of $\mathbf{B}_{\mathbf{2}} \times \mathbf{B}_{\mathbf{2}}$ is a subalgebra of $\mathbf{A}$.

The proof for De Morgan 0-algebras is again analogical, except instead of the nonstandard expansions of $\mathbf{B}_{\mathbf{2}}$ and $\mathbf{B}_{\mathbf{2}} \times \mathbf{B}_{\mathbf{2}}$ we may have to take the non-standard expansions of the extensions of these De Morgan lattices by an extra top and bottom element.

We conclude this section by exhibiting an infinite decreasing chain of standard quasivarieties of (regular non-idempotent) De Morgan 0-lattices. Consider, for $n \geq 1$, the quasiequation

$$
a_{1} \leq-a_{1}, \ldots, a_{n} \leq-a_{n}, a_{1} \vee \ldots \vee a_{n}=0 \Rightarrow x=y
$$


This quasiequation states that 0 cannot be expressed as a disjunction of $n$ or less elements of the form $a \wedge-a$. Clearly $\mathbf{A}$ is non-standard if and only if it satisfies $\left(\alpha_{n}\right)$ for each $n \geq 1$, hence the class of non-standard De Morgan 0-lattices is a quasivariety. The quasiequations $\left(\alpha_{n}\right)$ coincide with the quasiequations $\left(\beta_{n}\right)$ used by Gaitán and Perea in [5] if we restrict to the variety of De Morgan 0-lattices defined by $x \leq 0$.

Lemma 4.10. If $\mathbf{A}$ is a standard De Morgan 0-lattice which is generated as a De Morgan lattice by the finite set $X \subseteq \mathbf{A}$, then $0=\bigvee\{x \wedge-x \mid x \in X\}$.

Proof. It suffices to prove that for each term $t$ and each tuple $\bar{x}$ of elements from $X$ we have $t(\bar{x}) \wedge-t(\bar{x}) \leq \bigvee\{x \wedge-x \mid x \in X\}$. This is a straightforward proof by induction over the complexity of the term $t$.

Let $\mathbf{A}_{n}$ be the free De Morgan lattice on $n$ generators equipped with the standard inconsistency constant. Equivalently, $\mathbf{A}_{n}$ may be defined as the free distributive lattice on $2 n$ generators of the form $x_{i},-x_{i}$ for $1 \leq i \leq n$ with $0=\left(x_{1} \wedge-x_{1}\right) \vee \ldots \vee\left(x_{n} \wedge-x_{n}\right)$. For any generator $x_{i}$ of $\mathbf{A}_{n}$, let $h_{i}: \mathbf{A}_{n} \rightarrow \mathbf{D M}_{4}$ denote the unique homomorphism of De Morgan lattices such that $h_{i}(i)=\mathrm{b}$ and $h_{i}\left(x_{j}\right)=\mathrm{n}$ for all generators $x_{j}$ other than $x_{i}$, where $b$ and $n$ denote the two fixpoints of De Morgan negation on $\mathbf{D M}_{\mathbf{4}}$.

Lemma 4.11. The algebra $\mathbf{A}_{n}$ is regular and non-idempotent.

Proof. The non-idempotence of $\mathbf{A}_{n}$ is witnessed by any $h_{i}$, as $h_{i}(1)=\perp \not \subset=h_{i}(0)$. To prove that $\mathbf{A}_{n}$ is regular, suppose that $a \not \leq 0$. Then there is a homomorphism $h: \mathbf{A}_{n} \rightarrow$ $\mathbf{D M}_{4}$ such that $h(a) \not \subset h(0)$. We wish to show that $h(1 \wedge a) \not \leq h(0)$. Clearly $h(0)<\mathrm{T}$ and if $h(0)=\perp$, then $h(1 \wedge 0)=h(0) \not \leq h(0)$, hence $1 \wedge a \not \leq 0$. Suppose therefore without loss of generality that $h(0)=\mathrm{b}$. Since $h(0)=\mathrm{b}$, there is no $x_{i}$ such that $h\left(x_{i}\right)=\mathrm{n}$. Therefore $h(a)=\mathrm{\top}$ and there is some $x_{j}$ such that $h\left(x_{j}\right) \in\{\perp, \top\}$, hence $h(1)=\mathrm{\top}$ and $h(1 \wedge a) \not \leq h(0)$.

Lemma 4.12. The algebra $\mathbf{A}_{n}$ satisfies $\left(\alpha_{m}\right)$ if and only if $m<n$.

Proof. By Lemma 4.10, the inconsistency constant of $\mathbf{A}_{n}$ is a disjunction of $n$ elements. Vice versa, suppose that in $\mathbf{A}_{n}$, the inconsistency constant is a disjunction of $a_{i} \wedge-a_{i}$ for $1 \leq i \leq m$. Then there is some generator $x_{j}$ such that none of the elements $a_{i}$ belong to the subalgebra generated by $x$. We wish to show that $x_{j} \wedge-x_{j} \not \leq\left(a_{1} \wedge-a_{1}\right) \vee \ldots \vee\left(a_{m} \wedge-a_{m}\right)$.

If the term $t$ does not contain $x_{j}$, then $h_{j}(t)=\mathrm{n}$, and if the term $t$ does not contain any variable other than $x_{j}$, then $h_{j}(t)=\mathrm{b}$. It follows that if the term $t$ does not belong to the subalgebra generated by $x_{j}$, then $h_{j}(t) \neq \mathrm{b}$. Therefore, $h_{j}\left(x_{j} \wedge-x_{j}\right) \not \leq h_{j}\left(a_{1} \wedge-a_{1}\right) \vee \ldots \vee$ $h_{j}\left(a_{m} \wedge-a_{m}\right)$.

Proposition 4.13. The class of all standard De Morgan $\mathcal{J}$-lattices (0-lattices) is not an elementary class.

Proof. We show that these classes are not closed under ultraproducts. Let $\mathbf{A}$ be a nonprincipal ultraproduct of the standard algebras $\mathbf{A}_{i}$. Lemma 4.12 implies that $\mathbf{A}$ satisfies $\left(\alpha_{n}\right)$ for each $n \geq 1$. In other words, $\mathbf{A}$ is a non-standard De Morgan 0-lattice. The associated De Morgan $\mathcal{J}$-lattice, which is an ultraproduct of the standard De Morgan $\mathcal{J}$ lattices associated to the De Morgan 0-lattices $\mathbf{A}_{i}$, is therefore also non-standard. 
Theorem 4.14. There is an infinite decreasing chain of standard quasivarieties of (regular non-idempotent) De Morgan 0-lattices.

Proof. Take the quasivarieties generated by the sets of standard algebras $\left\{\mathbf{A}_{n} \mid n \geq i\right\}$ for $i \geq 1$. These generate standard quasivarieties of regular non-idempotent De Morgan 0 -lattices. Moreover, these quasivarieties are distinct, as witnessed by the quasiequations $\left(\alpha_{n}\right)$.

We do not know know many standard quasivarieties of De Morgan 0-lattices there are. Since totally inconsistent De Morgan 0-lattices are clearly termwise equivalent to De Morgan algebras, we know that there is a continuum of quasivarieties of De Morgan 0lattices which satisfy the equation $x \wedge-x \leq y \vee-y$. However, no non-trivial standard De Morgan 0-lattice which satisfies this equation can be totally inconsistent, therefore this result tells us nothing about standard quasivarieties of De Morgan 0-lattices.

\section{Conclusion}

We have succeeded in the task of pinpointing just how much quasiequational expressive power the standard inconsistency predicate adds to De Morgan lattices: the only new properties which were not expressible quasiequationally in the language of De Morgan lattices are regularity, its conjunction and disjunction with non-idempotence, and total inconsistency.

We have also seen that the only quasiequational expressive gain of adding a standard inconsistency constant (as opposed to a standard inconsistency predicate) to Kleene lattices consists in being able to define the class of idempotent Kleene lattices quasiequationally.

However, it remains an open question to determine how many standard quasivarieties of De Morgan lattices with an inconsistency constant there are. We have only managed to show that there are infinitely many.

Another natural open question is the following: is the lattice of standard quasivarieties a sublattice of the lattice of all quasivarieties of De Morgan lattice with an inconsistency constant? Equivalently, is the intersection of two standard quasivarieties necessarily standard?

\section{Acknowledgements}

This research was supported by the research program Progres Q14 Rationality Crisis and Modern Thought. The author is grateful to the referees for their comments which helped to improve the paper. 


\section{References}

[1] Michael E. Adams and Wiesław Dziobiak. Lattices of quasivarieties of 3-element algebras. Journal of Algebra, 166: 181-210, 1994.

[2] Nuel D. Belnap. A useful four-valued logic. In J. Michael Dunn and George Epstein, editors, Modern uses of multiple-valued logic, volume 2 of Episteme, pages 5-37. Springer Netherlands, 1977.

[3] Nuel D. Belnap and Joel Spencer. Intensionally complemented distributive lattices. Portugaliae Mathematica, 25: 99-104, 1966.

[4] J. Michael Dunn. Intuitive semantics for first-degree entailments and 'coupled trees'. Philosophical Studies, 29(3): 149-168, 1976.

[5] Hernando Gaitán and Milton H. Perea. A non-finitely based quasi-variety of De Morgan algebras. Studia Logica, 78: 237-248, 2004.

[6] Viktor A. Gorbunov. Algebraic Theory of Quasivarieties. Siberian School of Algebra and Logic. Springer, 1998.

[7] Alexej P. Pynko. Implicational classes of De Morgan lattices. Discrete Mathematics, 205(1-3): 171-181, 1999. 